ods (such as perturbation theory) which do not employ the Jacobi identity.

${ }^{7}$ K. Johnson, Nucl. Phys. 25, 431 (1961); S. Okubo, Nuovo Cimento $44 \mathrm{~B}, 1015$ (1966); D. G. Boulware and S. Deser, Phys. Rev. 151, 1278 (1966).

${ }^{8}$ For a different approach, see T. Das, V. S. Mathur, and S. Okubo, Phys. Rev. Letters 18, 761 (1967).

${ }^{9}$ The meson masses are taken from the latest compilation of A. H. Rosenfeld et al., Rev. Mod. Phys. $\underline{39}$, 1 (1967). The $K_{A}, A 1$, and $E$ are not definitely known to be $1^{+}$resonances, but in each case this is a plausible interpretation of the data. [There no longer seems to be any problem in understanding the $A 1$ width; J. Schwinger, Phys. Letters 24B, 473 (1967); H. Schnitzer and S. Weinberg, to be published.] If there are several mesons dominating a particular $\rho_{\alpha \alpha}{ }^{(0)}\left(\mu^{2}\right)$, then our predicted value for $F_{n}$ really gives the rms value of the corresponding $F_{n}$ 's.

${ }^{10}$ Only one of the $J_{\alpha}{ }^{\mu}$ can couple to any given meson; so it is not necessary to keep a label $\alpha$ on $F_{n}$.

${ }^{11} \mathrm{~K}$. Kawarabayashi and M. Suzuki, Phys. Rev. Letters 16, 255 (1966); Riazuddin and Fayyazuddin, Phys. Rev. 147, 1071 (1966); F. J. Gilman and H. J. Schnitzer, Phys. Rev. 150, 1362 (1966); J. J. Sakurai, Phys. Rev. Letters 17, 552 (1966); M. Ademollo, Nuovo Ci- mento $46 \mathrm{~A}, 156$ (1966). This estimate is based on several questionable approximations (for a discussion, see D. A. Geffen, to be published), but it does seem to work well.

${ }^{12}$ For a review of the experimental determination of $F_{K} / F_{\pi}$, see the rapporteur's talk by N. Cabbibo, in Proceedings of the Thirteenth International Conference on High Energy Physics, Berkeley, California, 1966 (University of California Press, Berkeley, 1967), p. 29. We shall show in a separate article that $\mathrm{SU}(3)$ symmetry breaking reduces the $K \rightarrow e+\nu+\pi$ decay amplitude by about $10 \%$, so that $\tan \theta$ is about $10 \%$ larger than had been thought, and the "experimental" value of $F_{K} / F_{\pi}$ is about 1.15 rather than 1.28 .

${ }^{13}$ J. Schwinger, Ann. Phys. (N.Y.) 2, 407 (1957); M. Gell-Mann and M. Levy, Nuovo Cimento 16, 705 (1960).

${ }^{14}$ The predictions of this model for scalar and pseudoscalar masses and coupling constants will be discussed in a separate article by S. Glas how and S. Weinberg, to be published.

${ }^{15}$ If we suppose that the average mass contributing to the $\Delta S=1$ vector spectral function is $825 \mathrm{MeV}$ rather than $890 \mathrm{MeV}$, then the prediction (11) becomes $F_{\kappa} / F_{\pi}$ $\cong 0.5$, and (13) becomes $\left|G_{\kappa N \Lambda} / G_{\pi N N}\right| \simeq 0.15$.

\title{
ERR A T A
}

$\bar{p} p$ ELASTIC SCATTERING FOR INCIDENT MOMENTA BETWEEN 1.0 and $2.50 \mathrm{BeV} / c$. B. Barish, D. Fong, R. Gomez, D. Hartill, J. Pine, and A. V. Tollestrup [Phys. Rev. Letters 17, 720 (1966)].

A mistake on the verticle scale of the graph in Fig. 1 was made. The scale should be multiplied by $\frac{1}{2}$. We are indebted to Ling-Lie Wang for this discovery.

SATURATION OF SUPERCONVERGENCE RELATIONS AND CURRENT-ALGEBRA SUM RULES FOR FORWARD AMPLITUDES. Frederick J. Gilman and Haim Harari, [Phys. Rev. Letters 18, 1150 (1967)].

A square root sign was dropped due to a typographical error in the sentence following Eq. (8), which should read: "where $f_{\pi}=135 \mathrm{MeV}$ is the decay constant of the charged pion, predicted by PCAC to satisfy $f_{\pi}=(2)^{1 / 2} G_{A} m_{N} g_{\pi N}$." 